\title{
Gossypiboma - the retained surgical swab: An enduring clinical challenge
}

\author{
R Naidoo, FCS (SA), MMed (Surgery); B Singh, FCS (SA), MD \\ Department of Surgery, Nelson R Mandela School of Medicine, University of KwaZulu-Natal, Durban, South Africa
}

Corresponding author: R Naidoo (ruvashni@gmail.com)

\begin{abstract}
Retained abdominal swabs remain a difficult problem. This review highlights the risk factors and index pathology, as well as markers that raise clinical suspicion, of a condition that may be elusive in presentation on account of its otherwise nonspecific signs and symptoms. A review of the English literature reporting retained abdominal swabs between 1992 and 2012 revealed 100 cases. Fifty-six percent of patients presented with pain, most commonly coupled with an abdominal mass or symptoms of bowel obstruction; $6 \%$ of patients presented with a fistula or a sinus; and $6 \%$ presented with extrusion of the swab; only $7 \%$ presented with signs indicative of sepsis. The most common initial surgery was obstetric and gynaecological (in $44 \%$ of cases); the second most common was general surgery (36\%), most commonly following cholecystectomy. Plain abdominal X-ray was done in $45 \%$ of patients, followed by ultrasound, computed tomography (CT) scan or both. CT scan is the best preoperative diagnostic tool currently. The varying presentations exhibited by this postsurgical entity will continue to perplex the attendant practitioner. Clinical suspicion assisted by ultrasound and CT scan will improve definitive diagnosis. While there are many checkpoints to prevent this rare yet significant complication, human error and the unpredictability of surgery may make elimination impossible. The challenges presented with a retained swab, although rare, will persist, and with it the devastating consequences for both patient and clinician. Because of this, especially in the era of a litigious mindset, surgical vigilance and pre-emptive measures cannot be emphasised enough.
\end{abstract}

S Afr J Obstet Gynaecol 2016;22(1):29-32 DOI:10.7196/SAJOG.2016.v22i1.1052

The term 'gossypiboma' is used to describe a retained surgical swab following any surgical procedure. Although a relatively rare occurrence, it has been noted since the beginning of surgical practice and continues to date, despite advances in operative theatre practice. The consequences of a retained swab can be life-altering or even fatal. The myriad of clinical presentations often mimic other clinical entities, making the diagnosis difficult.

\section{Epidemiology}

Accurate incidence of gossypiboma is hampered by the reluctance to report these cases, as well as the confidentiality agreements following legal settlements. ${ }^{[1]}$ This complication may sometimes remain asymptomatic, being discovered many years later or diagnosed at a different institution, and these factors impact on the accuracy of its incidence. The most common retained foreign body is the surgical swab, probably because of its frequent use, small size and being easily missed when blood soaked. ${ }^{[1]}$ The most common site involved is the abdominal cavity followed by the thoracic cavity ${ }^{[1]}$

For this review on the challenges presented by a retained swab, the search strategy and data collection were based on the review of published literature (Fig. 1).

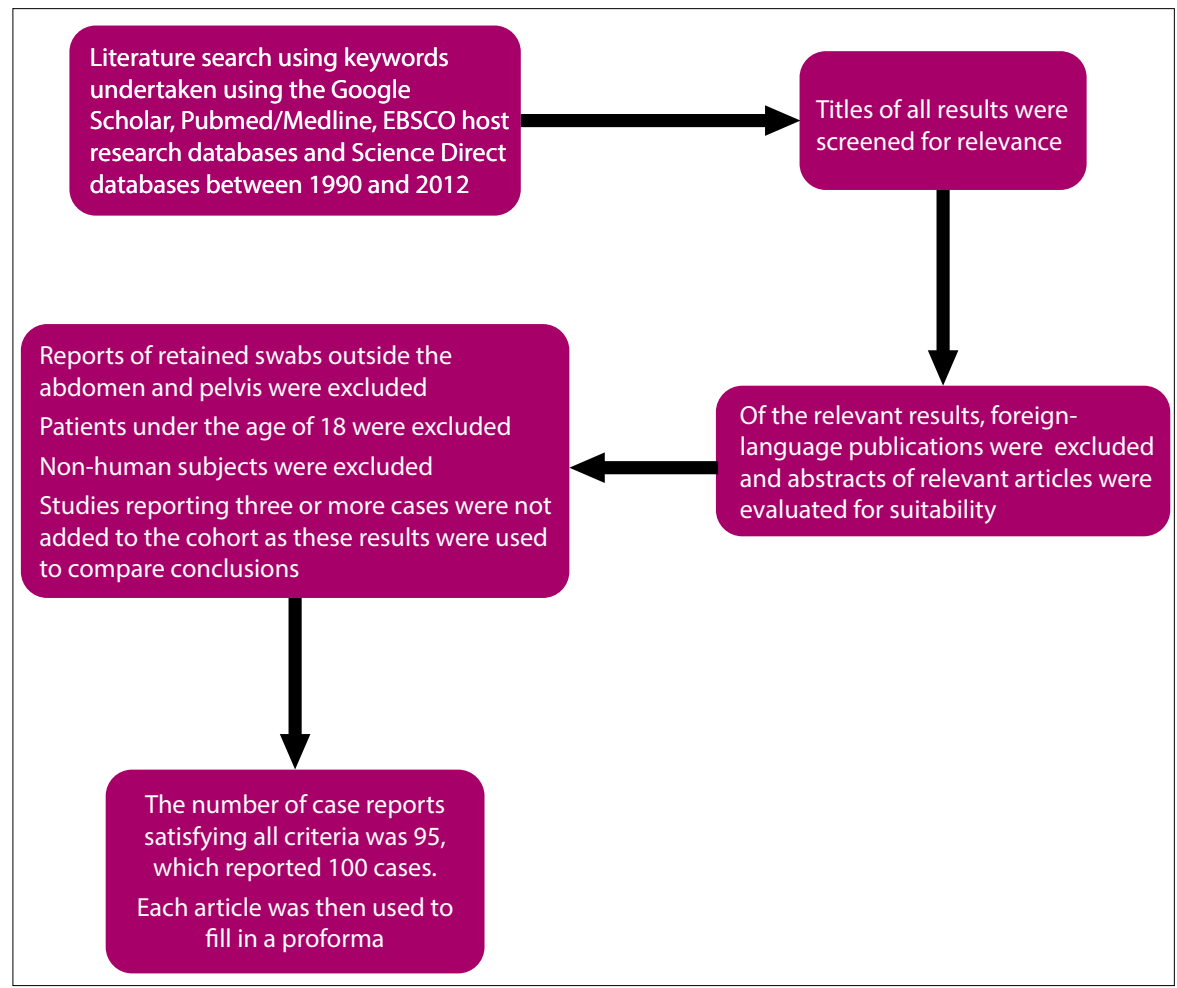

Fig. 1. Search strategy and data collection methods.

\section{Data abstraction and analysis}

The content from the literature was reviewed by a single author to eliminate bias in extracting information. The information was quantified under the following headings: clinical presentation, radiological imaging, initial surgery and time between surgery and presentation. Descriptive data analysis was conducted. 


\section{Ethical approval}

Ethical approval was sought and obtained from the University of KwaZulu-Natal Biomedical Research and Ethics Committee.

\section{Results}

There were 95 case reports deemed suitable for analysis, and these reported 100 cases of gossypiboma.

\section{Clinical presentation}

Table 1 lists case data. Pain was the most common presenting system; $56 \%$ of patients complained of severe pain as part of their presenting symptom complex but only $22 \%$ complained of isolated pain. Pain was usually coupled with one of the other common symptoms, such as an abdominal mass or symptoms of vomiting and/or constipation, which were considered as obstructive symptoms. These findings are consistent with the pathogenesis of the foreign body being walled off, with fibrosis creating a mass exerting external compression, or the foreign body migrating into the lumen to cause luminal obstruction.

Seven percent presented with signs of abdominal sepsis, suggesting that an

Table 1. Case data of reported cases of retained abdominal swabs $(N=100)$

\begin{tabular}{ll}
\hline & $\%$ \\
\hline Clinical presentation & 56 \\
Pain & 32 \\
Mass & 20 \\
Obstructive symptoms & 6 \\
Fistula or sinus & 7 \\
Sepsis & 6 \\
Extrusion & 40 \\
Other & \\
Surgical discipline & 36 \\
General surgery & 44 \\
Obstetrics and gynaecology & 6 \\
Trauma surgery & 10 \\
Urology & 4 \\
Other & \\
Radiological investigations & 45 \\
X-ray & 51 \\
Ultrasound & 64 \\
CT scan & 12 \\
MRI & 13 \\
Other &
\end{tabular}

For clinical presentation and radiological investigations, more than one parameter may apply per case; total may exceed $100 \%$. exudative pathological process is less common.

Only $6 \%$ of patients presented with a fistula, which underscores the remarkable way in which the body seals off the swab without allowing a loss in gastrointestinal continuity. Remarkably, $6 \%$ of patients also presented with complete extrusion of the swab via a normal orifice, which further amplifies the pathological processes outlined.

\section{Index surgical procedure}

The most common specialty predisposed to gossypiboma was obstetric and gynaecological surgery $(n=44,44 \%)$ (Table 1$)$. The most common operation performed was caesarean section $(n=21)$, which comprised $48 \%$ of the obstetric and gynaecological procedures performed, and abdominal hysterectomy $(n=13)$ comprising $30 \%$. The general surgery procedure that commonly predisposed to gossypiboma was open cholecystectomy $(n=19)$, comprising $53 \%$ of the total number of general surgery procedures.

\section{Radiological investigations}

Since the diagnosis is elusive, a range of investigations may have to be undertaken. Plain X-ray of the abdomen is the most basic investigation for abdominal complaints. Of the $45 \%$ of patients who had a plain abdominal X-ray, only $8 \%$ of patients required no further investigations, suggesting that plain X-ray may not be helpful in confidently diagnosing a gossypiboma. Abdominal ultrasound appears to be a valuable tool, with $21 \%$ of patients being successfully diagnosed with ultrasound and X-ray only; $64 \%$ of patients had a computed tomography (CT) scan.

\section{Discussion}

Retained abdominal swabs remain a difficult problem to eradicate even though tremendous strides in surgical practice have been made. Despite many advances, human error will always remain a variable that cannot be controlled; it is likely that this problem, although rare, will persist, and with it the devastating consequences for both patient and clinician. The purpose of this review was to highlight possible risk factors by looking at the index surgery, as well as to raise clinical suspicion of the diagnosis because of its nonspecific presentation, to ensure efficient diagnosis and management of this misfortune.
Limitations to the study included the reliance on reported data only, the sole use of electronic sources for the literature search and exclusion of literature prior to 1990, as well as exclusion of foreignlanguage publications. The study looked at reported cases only, which is biased towards more unusual cases.

The pathogenesis of a gossypiboma has been attributed to two types of foreignbody reactions which, between them, help to explain the variety of associated clinical presentations. The first type is an aseptic fibrous tissue reaction that involves fibroblast reaction, adhesion formation (resulting in either complete or incomplete encapsulation) and granuloma formation. ${ }^{[2]}$ The second type is an exudative inflammatory response which results in abscess formation or chronic internal or external fistulae, which may eventually result in transmural migration. The inflammatory reaction elicited by the swab initiates a process of self-extrusion, because the human body has the capability to recognise the sponge as a foreign body and its instinct is to eliminate it. ${ }^{[3]}$ Extrusion may be external and occur via a sinus or the abdominal incision itself; alternatively, it may be internal by eroding and perforating a hollow viscus and then migrating into the viscus aided by peristalsis. When complete, the swab may be extruded. If the migration is incomplete, it may obstruct the viscus. ${ }^{[3]}$

Clinical manifestation shows vast variation which relates to the location of the material within the abdomen. ${ }^{[4]}$ The clinical presentation is dictated by the type of foreign-body reaction. The presentation may be acute and severe or chronic and vague. Some of the most common presenting symptoms are abdominal masses, fistulas or sinuses, intestinal obstruction, intraabdominal abscesses and pain. The septic complications may also be influenced by the degree of intra-abdominal spillage and contamination during the index surgery. ${ }^{[2]}$

An array of radiological investigations has been described in the investigation of patients with a gossypiboma. The reason for this is that the diagnosis is not easy, and the clinical presentation may mimic diseases involving any of the intra-abdominal organs. The investigations most commonly used include plain radiographs, ultrasound, CT scan and magnetic resonance imaging (MRI). The nonspecific appearance of a gossypiboma has resulted in this entity 
being mistaken for tumours of the retroperitoneum, pancreas and spleen, gastrointestinal stromal tumours, and hydatid cystic disease, as well as bezoars.

Although plain X-ray was the most commonly performed investigation, the results showed that the majority of patients needed further investigation. Patients may present after many years, which may explain the lack of evidence on plain film. ${ }^{[5]}$ The diagnosis of a gossypiboma may be made easily on plain film if the radio-opaque marker is intact; however, this may have disintegrated over time. It may also become bound or folded. Radio-opaque markers may also be misinterpreted as calcifications, intestinal contrast material or surgical clips. Even in optimal situations the plain X-ray showed a 10 $25 \%$ false-negative rate in experimental studies done on cadavers. ${ }^{[2]}$

The second most commonly used investigation is ultrasound. This is a cheap, non-invasive investigation which seems a suitable next step in the diagnostic algorithm. Ultrasound in the immediate postoperative period may also be limited by intestinal gas as a result of a postoperative ileus or a physiological response to surgery, and may also be limited by painful incision sites and dressings over the incisions. ${ }^{[2]}$ This appearance is not very specific and almost always mandates further imaging, either by CT scan or less commonly by MRI. The literature reviewed suggests that the CT scan is the most consistent in making a preoperative diagnosis of a retained swab; in keeping with this, $64 \%$ of patients reviewed had a CT scan. The literature reviewed does offer some consistent appearance in the common investigations, despite the findings often being nonspecific (Table 2).

Table 2. Common features of a gossypiboma on plain X-ray, ultrasound and CT scan ${ }^{[15]}$

X-ray

\section{Radio-opaque mass}

Radio-opaque string-like appearance

Calcified mass

Mottled or sieve-like mass (may also be mistaken for faecal matter)

Features of intestinal obstruction such as dilated loops of bowel, air fluid levels and a paucity of air in the rectum

Ultrasound

Encapsulated complex cystic mass

Solid mass - hypoechoeic and heterogenous with dense acoustic shadows

CT scan

Thick-walled enhancing mass

Fluid and air bubbles trapped within the mass

A foreign metallic body may be seen within the mass (radio-opaque marker)

Calcified mass

Spongiform mass

Fat stranding

Prevention is always deemed better than cure. The first report of a retained swab was by Wilson in $1884 .{ }^{[6]}$ Since then, there has been constant development of techniques and protocols to decrease its incidence. Despite many innovations, human error cannot be completely eliminated.

One of the earliest strategies for prevention was the counting of swabs. This has become the standard of care in theatre practice. The counting is done when swab packs are opened and when the surgical wound is being closed. In some countries a routine double count is done at the end of an operation. This is the case in South Africa (SA). Each count should also be done by two theatre personnel. ${ }^{[7]}$ Multiple counts intraoperatively have also been advocated. Rotating nurses and scrub nurses is also advised to record all swabs placed inside the body, for haemostasis or exposure, while the surgery is taking place.

The routine checking of the body cavity by the surgeon, specifically checking for retained foreign bodies, is held to be one of the best ways of guarding against this problem. ${ }^{[7]}$

Radio-opaque marked swabs are used almost routinely in theatre practice today. ${ }^{[7]}$ They may be helpful when a swab count is incongruent. In this scenario (as opposed to the belated situation), radio-opaque markers are helpful in identification of retained swabs using plain X-ray. An X-ray is taken on table and reviewed prior to wound closure. Pitfalls of this method include incorrect positioning because of theatre equipment obstructing proper positioning.

The labelling of individual swabs with numbers or letters may assist in counting as it is more likely that a missing swab will be noticed if the numerical or alphabetic sequence is interrupted.

Poor communication and an imbalance of power between the scrub nurse and the surgeon may also lead to the nurse not alerting the surgeon of an incongruent swab count. The nurse may fear the wrath of the doctor for causing a delay in the conclusion of the operation. It has been suggested that equal accountability for mishaps and an improvement in relations may remedy this problem. ${ }^{[7]}$

Time management has been implicated in the omission of proper swab-counting protocol, as well as a lack of staff. The strain on staff to complete multiple emergency surgeries with no rest is great, although not an excuse for poor theatre practice. The only solution to this problem is fewer operations or more staff and equipment to run more theatres, which is not an easy-to-fix situation. ${ }^{[1]}$

A recent development is radio-nucleotide marked swabs. A gamma counter is used to locate lost swabs. By sweeping the operative field with a gamma counter, a swab within $5 \mathrm{~cm}$ of the counter can be excluded. This will mean that the missing swab is unlikely to be in the patient, and the search for the swab can continue within the theatre while the surgeon completes the

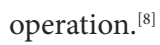

Electronic article surveillance, known as magnetomechanical technology that is widely used in the prevention of shoplifting, has also been applied to the counting of swabs. The swabs are tagged. At the end of the surgery an electronic detection device is passed over the operative field set up to detect the signal emitted by the tags, and should trigger the alarm if the tag which is attached to a swab is still in the body cavity. This method is still very much in the experimental phase, and carries the possibility of mechanical failure. ${ }^{[9]}$

Another risk factor implicated in swab retention is emergency surgery with multiple surgical teams and unexpected intraoperative changes. ${ }^{[1]}$ In this case a routine postoperative on-table X-ray may be prudent - and more cost-effective than a lawsuit. ${ }^{[10]}$

Higher risk is also associated with a change in nursing team midprocedure because of shift changes. ${ }^{[1]}$ Some hospitals prohibit this practice and compensate the nursing staff either monetarily or by repaying time. This practice varies between institutions in SA.

Surgical challenges such as obesity, haemorrhagic operations and difficult-to-reach anatomical areas, e.g. the pelvis, have been implicated as risk factors. ${ }^{[1,12]}$ This is in keeping with the study 
findings of obstetric and gynaecology index procedures being more common. The high number of mishaps in this group of patients can be related to the fact that a caesarean section tends to be an emergency operation. The increased risk during gynaecological procedures may be due to the technical difficulty of working in a deep, small cavity, where a bloodied swab may be easily missed. In this study, however, the most common general surgery procedure resulting in a retained swab was the cholecystectomy, which is an elective procedure. A possible explanation for this may be the limited operating space and the use of packing swabs to improve exposure. Interestingly, our results revealed that only $6 \%$ of the index procedures were trauma related.

In the past the nobility of the medical profession and trust of patients in their physicians protected medical professionals from legal accountability for their professional conduct. The position in society held by doctors cemented the belief that doctors would not intentionally make decisions that could negatively affect the clinical condition of their patients. This blind faith has changed in current practice, and the fallibility of medical professionals is now recognised. This trend started in Western countries and has crept into developing societies as patients become more knowledgeable. There is a paucity of established laws pertaining to medical error.

Medical misdemeanours are viewed differently throughout the world. In some countries, such as Turkey and Italy, medical cases pertaining to gossypiboma are harshly judged. They are viewed as criminal offences and charges are commenced as manslaughter or personal injury charges. ${ }^{[13]}$ In other countries medical cases usually belong to the category of civil law. Civil law obligations are of two types: (i) law of contract; and (ii) law of tort. When a medical professional undertakes to treat a patient, an informal but legally valid contract is undertaken. The implied agreement is that the doctor will diagnose the patient's complaint and treat in the normal manner according to generally accepted medical practice. A breach of this contract occurs when a clinician fails to provide the standard of care expected of any clinician with similar training and in similar circumstances. This failure may be viewed as at worst illegal or at best unethical. Tort is a civil wrong for which an action can be filed in court to recover damages for personal injury resulting from a negligent act. Medical negligence is recognised under the law of tort. When these cases are heard in court the onus is on the plaintiff (patient) to prove that the treatment provided by the medical professional did indeed cause injury or harm and was in fact negligent. This view tends to favour the medical professional. ${ }^{[3]}$

When medical negligence pertaining to a retained foreign object following surgery is taken to court in SA, the law seems to follow protocol set by the first reported case of Van Wyk v.
Lewis. The findings in this case indicated that the mere fact that the swab was left in situ does not imply negligence, because the circumstances surrounding the case should be taken into account. The case found in favour of the defendant, citing difficult intraoperative conditions with a medically unstable patient, thus not allowing for extended operative time under anaesthesia. ${ }^{[14]}$ SA seems to have a much more lenient stance than some other countries, especially in the developed world, where resources and working conditions play less of a role. However, this may change as patients in the developing world become cognisant of global trends and their rights.

Declarations. No financial aid was sought or received to support this work. This study was part of a thesis submission by Ruvashni Naidoo to the University of KwaZulu-Natal for the degree of Master of Medicine (Surgery).

Author contributions. BS conceptualised the project, provided supervision and critically reviewed the manuscript. RN was responsible for the literature search, data collection and manuscript writing. Both authors have read and approved the final manuscript.

\section{References}

1. Lincourt AE, Harrell A, Cristiano J, et al. Retained foreign bodies after surgery. J Surg Res 2007;138(2):170-174. DOI:10.1016/j.jss.2006.08.001

2. Sakorafas GH, Sampanis D, Lappas C, et al. Retained surgical sponges: What the practicing clinician should know. Langenbecks Arch Surg 2010;395(8):1001-1007. DOI:10.1007/s00423 010-0684-4

3. Gupta RL. The Medicolegal Aspects of Surgery. Delhi: Jaypee Brothers Medical Publishers, 1999:46-176.

4. Yildrim S, Tarim A, Nursal T, et al. Retained surgical sponge (gossypiboma) after intraabdominal or retroperitoneal surgery: 14 cases treated at a single center. Langenbecks Arch Surg 2006;391(4):383385. DOI:10.1007/s00423-005-0581-4

5. Caprio F, Lanza R, Amoroso L, et al. CT findings of surgically retained sponges and towels (gossypibomas). Eur Radiol 1993;3(4):383-385. DOI:10.1007/BF00167476

6. Wan W, Le T, Riskin L, et al. Improving safety in the operating room: A systematic literature review of retained surgical sponges. Curr Opin Anesthesiol 2009;22(2):207-214. DOI:10.1097/ ACO.0b013e328324f82d

7. Armstrong S. Improving the quality of care - learning through case studies. Retained abdominal swab. Professional Nursing Today 2008;12(2):7-10.

8. Bonicelli S, Citti P, Del Re E. Real time detection and tracking of gauzes by RFID UWB technique. 2010 IEEE International Conference on RFID. Orlando, FL: IEEE, 2010:97-101. DOI:10.1109/ RFID.2010.5467260

9. Fabian CE. Electronic tagging of surgical sponges to prevent their accidental retention. Surgery 2005;137(3):298-301. DOI:10.1016/j.surg.2004.10.003

10. Dossett LA, Dittus RS, Speroff T, et al. Cost effectiveness of routine radiographs after emergent open cavity operations. Surgery 2008;144(2):317-321. DOI:10.1016/j.surg.2008.03.012

11. Lauwers PR, van Hee RH. Intraperitoneal gossypibomas: The need to count sponges. World J Surg 2000;28(2):109-115. DOI:10.1007/s002689910084

12. Bani-Hani KE, Gharibeh KA, Yagha RJ. Retained surgical sponges (gossypiboma). Asian J Surg 2005;28(2):109-115.

13. Ulucay T, Dizdar MG, SunayYavuz M, et al. The importance of medico-legal evaluation in case with intraabdominal gossypiboma. Forensic Sci Int 2010;198(1-3):e15-e18. DOI:10.1016/j. forsciint.2010.01.013

14. Carstens PA, Pearmain D. Foundational Principles of South African Medical Law: Durban: LexisNexis, 2007.

15. Manzella A, Filho PB, Albuquerque E, et al. Imaging of gossypibomas: pictorial review. AJ R 2009;193(6 suppl):S94-S101. DOI:10.2214/AJR.07.7132 